\title{
Evaluation of Oxidative Stress and Antioxidant Level of Stroke Patients in Osun State, South-Western Nigeria
}

\author{
I. Akinlua*, M. F. Asaolu, 0. C. Ojo, G. O. Oyebanji \\ Biochemistry Department, Ekiti State University, Ado Ekiti, Nigeria \\ Email: ^ibikunle.akinlua@eksu.edu.ng
}

How to cite this paper: Akinlua, I., Asaolu, M.F., Ojo, O.C. and Oyebanji, G.O. (2019) Evaluation of Oxidative Stress and Antioxidant Level of Stroke Patients in Osun State, South-Western Nigeria. Journal of Biosciences and Medicines, 7, 189-194. https://doi.org/10.4236/jbm.2019.75020

Received: March 27, 2019

Accepted: May 24, 2019

Published: May 27, 2019

Copyright $\odot 2019$ by author(s) and Scientific Research Publishing Inc. This work is licensed under the Creative Commons Attribution International License (CC BY 4.0).

http://creativecommons.org/licenses/by/4.0/

(c) (i) Open Access

\begin{abstract}
Stroke is a leading cause of morbidity and mortality worldwide. Oxidative stress which is as a result of increased activity of free radicals and decreased antioxidant mechanism plays a major role in the pathogenesis of stroke. This study determined the plasma level of malondialdehyde (MDA), a marker of lipid peroxidation and oxidative stress and enzymatic Antioxidants namely, superoxide dismutase (SOD), Glutathione peroxidase (GPx), and Catalase (CAT) in sixty stroke patients of both sexes attending Wesley Guide Hospital, Obafemi Awolowo University Teaching Hospital, Ilesa, Osun State. An equal number of healthy subjects were used as control. The result of this study revealed a significantly $(\mathrm{p}<0.05)$ high plasma level of MDA in stroke patients when compared with the control subjects. Plasma level of SOD in stroke patients was significantly higher $(\mathrm{p}<0.05)$ than the control subjects while plasma GPx and CAT level were found to be significantly lower $(\mathrm{p}<0.05)$ in stroke patients than the control subjects. The result of the study indicated high level of lipid peroxidation and oxidative stress in stroke patients and high degree of free radicals presence as indicated by the high level of SOD.
\end{abstract}

\section{Keywords}

Stroke, Malondialdehyde, Catalase, Superoxide Dismutase, Glutathione

Peroxidase, Lipid Peroxidation

\section{Introduction}

Stroke is a form of cardiovascular disease that decreases blood supply to the brain which results in rapid loss of brain function. This can be due to ischemia (lack of blood flow), or by blockage (thrombosis, arterial embolism) [1] or a hemorrhage. As a result, the affected area of the brain cannot function, which 
might result in an inability to move one or more limbs on one side of the body, inability to understand or formulate speech, or an inability to see one side of the visual field [2].

The symptoms experienced by stroke patient will depend on which part of the brain is affected. It is a medical emergency and can cause permanent neurological damage and death. Risk factors for stroke include old age, high blood pressure, previous stroke or transient ischemic attack (TIA), diabetes, high cholesterol, tobacco smoking and atrial fibrillation [3]. Stroke is the second leading cause of death worldwide and also the leading cause of acquired adult disability with high socio-economic impact [4].

Oxidative stress arises from an imbalance between the generation of reactive oxygen (ROS) and nitrogen species (RNS) and the antioxidant defence system in the body so that the latter become overwhelmed [5]. Antioxidant such as reduced glutathione (GSH), vitamin C, vitamin E, SOD, catalase (CAT), GSH peroxidases (GSH-Pxs), are present in cells to remove ROS and RNS. Oxidative stress is a subject of intensive investigation and is considered to be one of the mechanisms involved in neuronal damage due to ischemia/reperfusion in stroke patients [6].

Lipid peroxidation is a free radical related process, which is potentially harmful because its uncontrolled, self-enhancing process causes disruption of membranes, lipids and other cell components. A lot of oxygenated compounds, particularly aldehydes such as Malondialdehyde (MDA) are produced during the attack of free radicals to membranes, lipoprotein and polyunsaturated fatty acids [7]. Thus lipid peroxidation in the blood provides useful information for the prognosis of CVD patients. This imbalance will cause damage to cellular components and tissues in the body leading to oxidative stress and as well as the decrease in total antioxidant capacity.

This work evaluates the plasma levels of malondialdehyde (MDA), superoxide dismutase (SOD), catalase and glutathione peroxidase (GPx) in stroke patients and healthy subjects with a view to providing information on diagnosis and treatment.

\section{Methodology}

This study included 60 freshly diagnosed stroke patients at the outpatient department of Wesley Guide Hospital, Obafemi Awolowo University Teaching Hospital, Ilesa, Osun State, and the same number of normal healthy subjects, without any known disease. Blood pressure was taken on the left arm after 5 minutes' relaxation, in a sitting position, using a standard mercury sphygmomanometer with appropriate cuff size; systolic (SBP) and diastolic (DBP) blood pressures corresponded to Korotkoff sounds I and V, respectively. The average of three readings, taken at first visit, was used for further analysis. Height and body weight were measured with participants standing without shoes and heavy outer garments. $5 \mathrm{ml}$ of venous blood was collected into a heparin bottle and centrifuged. The plasma was collected and placed in another bottle containing 
no anticoagulant for analysis. The total amount of lipid peroxidation products present in the samples was estimated by the thiobarbituric acid (TBA) method which measured the malondialdehyde (MDA) reactive products according to the method of Ohkawa et al. (1979) [8]. Catalase activity was colorimetrically assayed as described by Sinha (1972) [9]. SOD determination was done by the method of Fukui et al. (2003). Glutathione peroxidase was measured by the method described by Rotruck et al. (1973) [10]. Statistical analysis of result was done using one-way analysis of variance; level of significant difference was set at $\mathrm{p}<0.05$. All patients gave their informed consent, and this study was approved by the Institutional Review Board of the Hospital.

\section{Discussion}

Stroke is a form of cardiovascular disease affecting blood supply to the brain, which is caused either by a blockage or a rupture in the vessel supplying blood to the brain. From the result of this work, as shown in Table 1, there is a significant increase $(\mathrm{p}<0.05)$ in the age and blood pressure of stroke patients respectively when compared with control subjects respectively which confirm the fact that high blood pressure (hypertension) and old age are factors in the development of stroke. This finding supports the report that age is the most important risk factor in developing cardiovascular or heart diseases [11]. It also correlates with data obtained from a study done on stroke patients in Northern Manhattan where the mean age was $68.8 \%$ [12]. Multiple explanations have been proposed to explain why age increases the risk of cardiovascular/heart diseases. One of them is related to serum cholesterol level. In most populations, the serum total cholesterol level increases as age increases [13]. The plasma level of MDA in stroke patient as shown in Table 2 is significantly higher $(\mathrm{p}<0.05)$ when compared with the control. MDA which is a breakdown product of lipid peroxidation has been found to increase in stroke patient [14] [15]. The significantly high level of MDA in stroke patients as revealed in this study agrees with the outcome of a study by Abdulkaldar et al. (2007) [16]. who reported a high MDA level in the serum of stroke patients when compared with the control group. This he attributed to the fact that cellular membranes in the brain are very rich in polyunsaturated fatty acids, which are especially sensitive to free radical attack. The result of the study in Table 2 reveals low Glutathione (GPx) activity in stroke patients when compared with the control. This was consistent with findings of Demirkaya et al. (2001) [17], who reported that GPx in the erythrocyte of patients with hemispheric ischemic stroke decreased significantly within $24 \mathrm{hrs}$ after the onset of stroke symptoms. The results also agree with the report of Seema et al. (2010) [18], who carried out a study on the role of antioxidants in cardiovascular diseases reported a decreased level of GPx in stroke patients and lschaemic heart disease patients The activity of SOD was significant $(\mathrm{p}<0.05)$ higher in stroke patient than the control subjects as shown in Table 2. The increase in the activity of SOD as revealed in this study consisted with the report of Gruener et al. (1994) [19]. 
Table 1. Parameters showing the anthropometric measurement of stroke patients and control subjects.

\begin{tabular}{ccc}
\hline Parameters & Control & Stroke Patients \\
\hline Age (YRS) & $24.50 \pm 1.63^{\mathrm{b}}$ & $61.0 \pm 13.28^{\mathrm{a}}$ \\
Weight (KG) & $53.66 \pm 22.6^{\mathrm{b}}$ & $83.5 \pm 17.52^{\mathrm{a}}$ \\
Height (M) & $1.48 \pm 0.2^{\mathrm{a}}$ & $1.62 \pm 0.20^{\mathrm{a}}$ \\
B/P (mm/HG) & $116.6 / 68.6^{\mathrm{b}}$ & $193.4 / 105.3^{\mathrm{a}}$ \\
BMI (KG/M $)$ & $24.5^{\mathrm{b}}$ & $31.6^{\mathrm{a}}$ \\
\hline
\end{tabular}

Results are presented as means \pm standard deviation. Values with different superscript are significantly different. B/P (Blood pressure), BMI (Body mass index).

Table 2. Parameters of blood plasma showing the mean malondialdehyde (MDA), catalase (CAT), superoxide dismutase (SOD), and glutathione peroxidase (GPx) value of stroke patients and control subjects.

\begin{tabular}{ccc}
\hline Parameter & Control & Stroke Patients \\
\hline SOD (U/L) & $206.95 \pm 30.23^{\mathrm{b}}$ & $217.3 \pm 16.11^{\mathrm{a}}$ \\
CAT (U/L) & $50.46 \pm 12.55^{\mathrm{a}}$ & $36.9 \pm 16.33^{\mathrm{a}}$ \\
GPx (pg/ml) & $533.3 \pm 180.9^{\mathrm{a}}$ & $186.91 \pm 48.82^{\mathrm{b}}$ \\
$\mathrm{MDA}(\mathrm{nmol} / \mathrm{L})$ & $1.98 \pm 0.42^{\mathrm{a}}$ & $6.32 \pm 1.36^{\mathrm{b}}$ \\
\hline
\end{tabular}

Results are presented as means \pm standard deviation. Values with different superscript are significantly different.

There is a significant decrease in the activity of catalase in the stroke patients when compared with the control subjects. The increase or decrease in the activity of antioxidant enzymes in stroke patients may result from the degree of damage by reactive oxygen species (ROS).

\section{Conclusion}

This study confirms the fact that high blood pressure is a major factor in the prognosis of stroke; it also reveals a possibly high level of free radicals and lipid peroxidation in stroke patients which are factors that could be monitored to control and manage this life threatening disease.

\section{Acknowledgements}

We appreciate the contributions of the entire staff of the outpatient department of Wesley Guide Hospital, Obafemi Awolowo University Teaching Hospital, Ilesa, Osun State.

\section{Conflicts of Interest}

The authors declare no conflicts of interest regarding the publication of this paper.

\section{References}

[1] Sims, N.R. and Muyderman, H. (2009) Mitochondria, Oxidative Metabolism and 
Cell Death in Stroke. Biochimica et Biophysica Acta, 1802, 80-91. https://doi.org/10.1016/j.bbadis.2009.09.003

[2] Donnan, G.A., Fisher, M., Macleod, M. and Davis, S.M. (2008) Stroke. The Lancet, 371, 1612-1623. https://doi.org/10.1016/S0140-6736(08)60694-7

[3] Giles, M.F. and Rothwell, P.M. (2008) Risk Prediction after TIA: The ABCD System and Other Methods. Geriatrics, 63, 10-16.

[4] Lozano, R., Naghavi, M., Foreman, K., Lim, S., Shibuya, K., Aboyans, V., Abraham, J., Adair, T., Aggarwal, R. and Ahn, S.Y. (2012) Global and Regional Mortality from 235 Causes of Death for 20 Age Groups in 1990 and 2010: A Systematic Analysis for the Global Burden of Disease Study 2010. The Lancet, 380, 2095-2128.

https://doi.org/10.1016/S0140-6736(12)61728-0

[5] Rodrigo, R., González, J. and Paoletto, F. (2011) The Role of Oxidative Stress in the Pathophysiology of Hypertension. Hypertension Research, 34, 431-440. https://doi.org/10.1038/hr.2010.264

[6] Sanderson, T.H., Reynolds, C.A., Kumar, R., Przyklenk, K. and Huttemann, M. (2013) Molecular Mechanisms of Ischemia-Reperfusion Injury in Brain: Pivotal Role of the Mitochondrial Membrane Potential in Reactive Oxygen Species Generation. Molecular Neurobiology, 47, 9-23. https://doi.org/10.1007/s12035-012-8344-Z

[7] Guéraud, F., Atalay, M., Bresgen, N., Cipak, A., Eckl, P.M. and Huc, L. (2010) Chemistry and Biochemistry of Lipid Peroxidation Products. Free Radical Research, 44, 1098-1124. https://doi.org/10.3109/10715762.2010.498477

[8] Ohkawa, H., Ohishi, N. and Yagi, K. (1979) Assay for Lipid Peroxides in Animal Tissues by Thiobarbituric Acid Reaction. Analytical Biochemistry, 95, 351-358. https://doi.org/10.1016/0003-2697(79)90738-3

[9] Sinha, A.K. (1972) Colorimetric Assay of Catalase. Analytical Biochemistry, 47, 389-394. https://doi.org/10.1016/0003-2697(72)90132-7

[10] Rotruck, J.T., Pope, A.L., Ganther, H.E., Swanson, A.B., Hafeman, D.G. and Hoekstra, W.G. (1973) Selenium: Biochemical Role as a Component of Glutathione Peroxidase. Science, 179, 588-590. https://doi.org/10.1126/science.179.4073.588

[11] Finegold, J.A., Asaria, P. and Francis, D.P. (2012) Mortality from Ischaemic Heart Disease by Country, Region, and Age: Statistics from World Health Organisation and United Nations. International Journal of Cardiology, 168, 934-945. https://doi.org/10.1016/j.ijcard.2012.10.046

[12] Willey, J.Z., Qiang, X., Albala, B.B., Myunghee, C.P., Moon, Y.P., Sacco, R.L. and Elkind, M.S.V. (2009) Lipid Profile Components and Risk of Stroke-A Northern Manhattan Study. Archives of Neurology, 66, 1400-1406.

https://doi.org/10.1001/archneurol.2009.210

[13] Jousilahti, V. and Tuomilehto, P. (1999) Sex, Age, Cardiovascular Risk Factors, and Coronary Heart Disease. Circulation, 99, 1165-1172. https://doi.org/10.1161/01.CIR.99.9.1165

[14] Sharpe, P.C., Mulholland, C. and Trinick, T. (1994) Ascorbate and Malondialdehyde in Stroke Patients. Irish Journal of Medical Science, 163, 488-491. https://doi.org/10.1007/BF02967089

[15] Imre, S.G., Fekete, I. and Farkas, T. (1994) Increased Proportion of Docosahexanoic Acid and High Lipid Peroxidation Capacity in Erythrocytes of Stroke Patients. Stroke, 25, 2416-2420. https://doi.org/10.1161/01.STR.25.12.2416

[16] Abdulkadr, Y., Dikan, K., Serap, Y., Recep, A. and Fatih, A. (2007) Increased Lipid Peroxidation and Decreased Antioxidant Response in Serum and Cerebrospinal 
Fluid in Acute Ischemic Stroke. Journal of Medical Sciences, 37, 75-81.

[17] Demirkaya, S., Topcuoglu, M.A., Aydin, A., Ulas, U.I., Isimer, A.I. and Vural, O. (2001) Malondialdehyde, Glutathione Peroxidase and Superoxide Dismutase in Peripheral Blood Erythrocytes of Patients with Acute Cerebral Ischemia. European Journal of Neurology, 8, 43-51. https://doi.org/10.1046/j.1468-1331.2001.00166.x

[18] Seema, L.J., Ujjwala, J.K., Vasant, T.S. and Deshmukh, Y.A. (2010) Role of Oxidants Andantioxidants in Patients with Cardiovascular Diseases. Asian Journal of Medical Sciences, 2, 181-184.

[19] Gruener, N., Gross, B., Gozlan, O. and Barak, M. (1994) Increase in Superoxide Dismutase after Cerebral Accident. Life Sciences, 54, 711-713.

https://doi.org/10.1016/0024-3205(94)90159-7 